\title{
Der säkulare Trend
}

Erhard Taverna
Der Gang in ein Museum zeigt es eindrücklich: Wir sind grösser als unsere Vorfahren. Ihre Betten wären viel zu knapp, und eine Ritterrüstung gehörte heute in die Abteilung für Kindergrössen. Grosswüchsige gab es immer, doch sie waren die bestaunten Ausnahmen ihrer Zeit. In allen Industriestaaten lässt sich über die letzten 12 bis 15 Generationen ein erstaunlicher Wandel nachweisen - nicht gleichmässig und auch nicht ohne Rückschläge, aber dennoch eine $\mathrm{Zu}$ nahme der Körpermasse, der Langlebigkeit und der Gesundheit. Diese Änderung wird als säkularer Trend bezeichnet und ist seit Mitte des 19. Jahrhunderts gut belegt. Der amerikanische Ökonom Robert Fogel, Nobelpreisträger 1993, und Co-Autoren haben dazu eine riesige Datenmenge ausgewertet und ihre Folgerungen publiziert: «The Changing Body. Health, Nutrition, and Human Development in the Western World since 1700.» Das Werk ist der vorläufige $\mathrm{Ab}$ schluss einer Jahrzehnte dauernden Forschung in minutiös gesammelten Quellen, die eine 300-jährige Entwicklung belegen. Die Ökonomen zeigen, dass anthropometrische Masse weit mehr über den individuellen Wohlstand verraten, als dies die üblichen Vergleiche von Bruttosozialprodukt und Pro-KopfEinnahmen erlauben. Der «biologische Lebensstandard» hat sich so massiv verändert, dass sie dafür den Ausdruck technophysio evolution geprägt haben, um

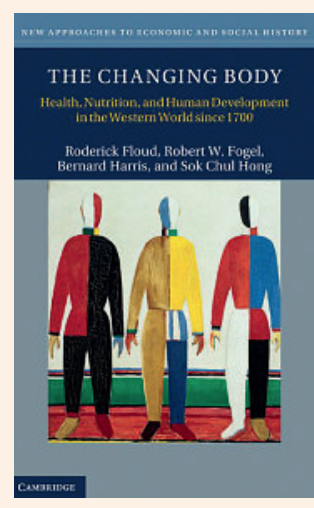

Roderick Floud, Robert W. Fogel, Bernard Harris, Sok Chul Hong The Changing Body. Health, Nutrition, and Human Development in the Western World since 1700 .

Cambridge: Cambridge University Press; 2011. 472 Seiten. Englisch. $34.50 \mathrm{CHF}$. ISBN 978-0-521-70561-5

des 18. Jahrhunderts zunehmend statistische Erhebungen der Behörden. Die Autoren errechnen die tägliche Kalorienaufnahme, berücksichtigen die Zusammensetzung der Ernährung, die Arbeitszeit und Produktivität der jeweiligen Gesellschaft. Aus der Subsistenz- entwickelte sich allmählich eine Überflussökonomie, gefördert durch neue Anbaumethoden wie den eisernen Pflug, den Pferde statt Ochsen zogen, Düngemittel und andere technische Errungenschaften sowie eine zunehmende Alphabetisierung.

Die Rechnung ist relativ einfach: Der Ernährungszustand einer Generation, ausgedrückt durch die

\section{Geht die Entwicklung ad infinitum weiter, wachsen Menschen in den Himmel, liegt ein Durchschnittsalter von 130 Jahren bei optimaler Versorgung im Bereich der Möglichkeit?}

die enorme Anpassung des menschlichen Körpers an die veränderten kulturellen Bedingungen hervorzuheben.

Weisse, männliche Amerikaner lebten während der Kolonialzeit länger als die Europäer und waren zudem $7 \mathrm{~cm}$ grösser, schrumpften aber zwischen 1860 und 1890 um durchschnittlich 4,4 cm. Ein Franzose in seinen 30ern brachte zu Revolutionszeiten gerade einmal $50 \mathrm{~kg}$ auf die Waage, im Vergleich zu den aktuellen $77 \mathrm{~kg}$. In Norwegen ist ein 22-jähriger Mann heute rund $14 \mathrm{~cm}$ grösser als 1850, in den Niederlanden, wo die grössten Menschen leben, waren die Wehrpflichtigen $199016 \mathrm{~cm}$ grösser als 1860. Zu den frühesten Aufzeichnungen gehören Chroniken aus Massachusetts ab 1654, dazu kommen Rekrutierungslisten, Angaben über soziale Werke und Kindersterblichkeit, Tauf- und Sterbebücher und ab Mitte
Körpermasse, bedingt die Lebenserwartung und das Arbeitspotential. Kräftigere Menschen produzieren mehr, damit stehen mehr Güter und Dienstleistungen zur Verfügung. Besser ernährte Mütter bringen gesündere Kinder zur Welt, die gegenüber Krankheiten resistenter und potentiell intelligenter sind. Vom erarbeiteten Wohlstand profitiert die nächste Generation, die ihrerseits wiederum die Ausgangbedingungen für ihre Kinder verbessert. Und so immer weiter, ad infinitum, wie die Autoren schreiben. Noch 1850 gingen 47\% der Todesursachen in den USA auf Infektionen zurück, 2005 waren es noch 1,8\%. Wie in Europa verbesserten sich die hygienischen Zustände Ende des 19. Jahrhunderts in den Städten dramatisch. Lebten früher die Menschen auf dem Lande länger, so war es allmählich umgekehrt. Die demographische Geschichte der Neuzeit wird hier buchstäblich auf 
den Leib geschrieben, ganz nach dem Motto des deutschen Philosophen Feuerbach «Der Mensch ist, was er isst.»

Geht diese Entwicklung wirklich ad infinitum weiter, wachsen Menschen in den Himmel, liegt ein Durchschnittsalter von 130 Jahren bei optimaler Versorgung im Bereich der Möglichkeit, wie die Autoren behaupten? Voraussetzung sind günstige sozioökonomische Bedingungen, was auch neuere Studien belegen, die Körpergrösse von Wehrpflichtigen des Jahrgangs 1971 in der DDR mit der von Wehrpflichtigen in der Bundesrepublik vergleichen. In den vergangenen 40 Jahren ist aber die Geburtslänge in allen Industrieländern inklusive Japan konstant geblieben. Der säkulare Trend hat sich in allen diesen Ländern verlangsamt, und seit den 1950er Jahren liegt das Menarchealter als weiterer Indikator des säkularen Trends bei konstanten 13 Jahren. In den USA ist sogar eine rückläufige Tendenz sichtbar. Kritiker werfen Fogel und seiner Forschergruppe vor, dass sie ihren Bericht zu einseitig auf die Ernäh-

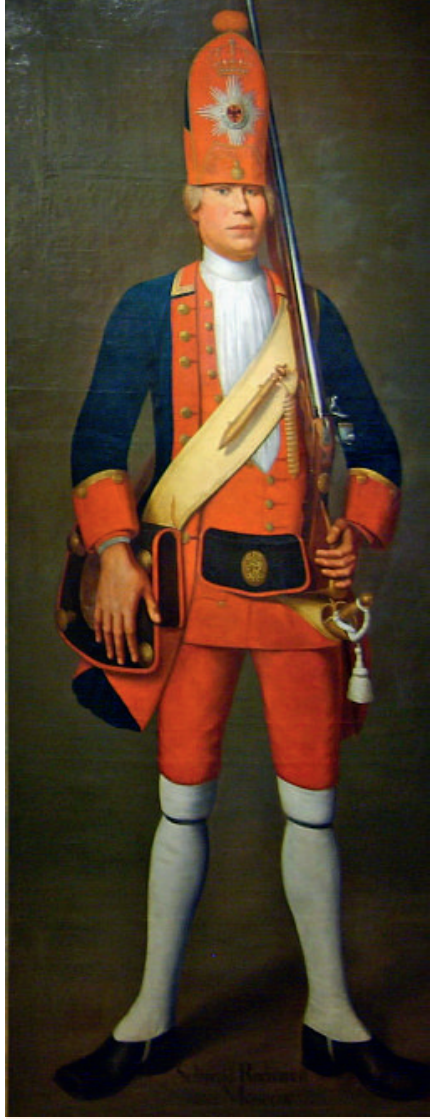

Die «Langen Kerls» des Königs in Preussen Friedrich Wilhelm I. (1688-1740) mussten mindestens 1,88 Meter gross sein - damals eine Besonderheit, heute nicht mehr. rung fokussieren. Tatsache ist, dass die Ursachen für den säkularen Trend komplex sind und viele biologische Mechanismen, wie Genetik, Epigenetik und möglicherweise Umweltfaktoren noch unverstanden bleiben. Gibt es Grenzwerte für Körpermasse als Anzeichen für das Umschlagen in einen Überlebensnachteil, wie die Waaler-Kurven nahelegen. Fogel et al. diskutieren diese Aspekte wie auch die Nachteile eines Überkonsums an Kalorien mit einer Zunahme von Krankheiten wie Übergewicht und Diabetes, sie dokumentieren auch, wie Sozialstatus, Geschlecht, Rasse und Ethnie in den USA die Indikatoren wieder zunehmend auseinanderdriften lassen. Wird Thomas Malthus, der britische Ökonom, der auch Charles Darwin beeinflusst hat, doch noch Recht bekommen, wächst die Erdbevölkerung schneller als die Landwirtschaft? «Will these improvements continue?», fragen die Buchautoren und antworten darauf ehrlich: «We do not know.»

Doch die Lektüre lohnt sich, die Fülle an historischen Dokumenten ist eindrücklich. Die Texte, Tabellen und grafischen Darstellungen sind für jeden an ökonomischen und medizinischen Fakten Interessierten eine wahre Fundgrube. 